\title{
Carnets
}

Revue électronique d'études françaises de l'APEF

Deuxième série - 8 | 2016

Du Français en cause aux causes du français

\section{Valoriser la spécificité du français face à la mutation du paradigme linguistique}

\section{Raymond Renard}

\section{(2) OpenEdition}

1 Journals

\section{Édition électronique}

URL : http://journals.openedition.org/carnets/1914

DOI : 10.4000/carnets. 1914

ISSN : 1646-7698

Éditeur

APEF

\section{Référence électronique}

Raymond Renard, «Valoriser la spécificité du français face à la mutation du paradigme linguistique », Carnets [En ligne], Deuxième série - 8 | 2016, mis en ligne le 30 novembre 2016, consulté le 01 mai 2019. URL : http://journals.openedition.org/carnets/1914; DOI : 10.4000/carnets. 1914

Ce document a été généré automatiquement le 1 mai 2019.

\section{(c) (i) (8)}

Carnets est mis à disposition selon les termes de la licence Creative Commons - Atribution - Pas d'utilisation commerciale 4.0 International. 


\title{
Valoriser la spécificité du français face à la mutation du paradigme linguistique
}

\author{
Raymond Renard
}

\section{Écologie des langues}

1 J'aimerais, ainsi que je l'ai annoncé dans mon résumé, situer d'abord ma réflexion sur le destin du français dans la problématique de l'écologie des langues.

2 Calvet nous en a, dès 1999, offert une vue cohérente. Les langues sont reliées entre elles par des bilingues, et les différents bilinguismes sont reliés en termes gravitationnels. Autour d'une langue hypercentrale (l'anglais) gravitent une dizaine de langues supercentrales (le français, l'espagnol, le russe, l'arabe, le chinois, le hindi, le malais, etc.) autour desquelles gravitent deux cents langues centrales qui sont à leur tour le pivot de la gravitation de quatre à cinq mille langues périphériques.

À chacun des niveaux de ce système peuvent se manifester deux tendances, l'une vers un bilinguisme horizontal (acquisition d'une langue de même niveau que la sienne) et l'autre vers un bilinguisme vertical (acquisition d'une langue de niveau supérieur), ces deux tendances étant le ciment du modèle. Les différentes langues étant des produits de la pratique sociale, on comprend aisément le poids sur les "tendances" de facteurs particulièrement linguicides comme l'urbanisation, le souci de promotion sociale, le type de scolarisation ou, comme je l'évoquerai bientôt, la pression d'un monolinguisme porté par les vagues d'une mondialisation uniformisante.

4 Toute politique linguistique a dû prendre en compte ces données, la nécessité d'appuyer la modernisation sur des langues de grande diffusion impliquant une grande vigilance devant le risque réel d'un colonialisme socioéconomique et culturel. L'aménagement linguistique suppose à l'évidence une intervention de l'Etat. Sans celle-ci, les langues et les cultures endogènes sont vouées à la loi du plus fort. Et c'est bien ce qui se passe. Le pluralisme linguistique et culturel est aujourd'hui en grave danger d'être laminé par le 
rouleau compresseur de l'homogénéisation culturelle par le marché, par le «tout économique». Cette homogénéisation réductrice est en train d'appauvrir dangereusement le patrimoine de l'humanité, au profit de l'anglo-américain et d'une culture matérialiste de masse, où se confondent culture et divertissement.

\section{Changement de paradigme}

5 Fort heureusement, le récent passage de millénaire nous a fait assister à un véritable sursaut de résistance que l'on peut qualifier de changement profond de paradigme linguistique.

6 En deux mots, en une décennie, trois organismes internationaux de poids y ont contribué, en abandonnant la logique de l'unité - celle de Rivarol, celle aussi du colonialisme - au profit de celle de la pluralité.

7 Coup sur coup, en effet, dans les premières années du siècle, l'Organisation Internationale de la Francophonie (OIF), l'Unesco et l'Union Européenne (UE) annoncent de véritables plans de bataille.

- OIF

8 En 2003, les Etats généraux de l'enseignement du français en Afrique subsaharienne francophone (600 participants), tenus à Libreville, créent la surprise en mettant l'accent sur « l'égalité des langues-cultures et le refus de toute hiérarchisation dans ce domaine» et en recommandant la mise en place d'un plurilinguisme fonctionnel qui associe au français la langue du milieu et une langue régionale.

9 Dans son discours de clôture très attendu, le Secrétaire général de l'OIF, Abdou Diouf, exprime sa détermination en des termes forts :

Quarante ans après les indépendances africaines, la dégradation des systèmes éducatifs d'Afrique s'aggrave et il est temps de lui trouver des solutions. Celle qui consiste à associer davantage les langues natales et la langue française arrive à maturité. Elle est essentielle. [...] il est donc indispensable, dans un premier temps, «d'installer confortablement l'enfant dans sa langue maternelle » pour l'ouvrir, ensuite, sur une langue française désormais « partenaire » des langues-cultures [..] en contact.

$10 \mathrm{Au}$ début $\mathrm{du} \mathrm{xxI}^{\mathrm{e}}$ siècle, en matière linguistique, la Francophonie passait enfin de l'apartheid au partenariat.

- UNESCO

11 La même année, l'Unesco, à l'occasion du cinquantenaire de son célèbre Rapport de 1953, rappelle en un document important publié sous le titre L'éducation dans un monde multilingue (Unesco, 2003) les 3 principes de base à partir desquels sont développées ses orientations plus spécifiques :

1. L'Unesco encourage l'enseignement dans la langue maternelle en tant que moyen d'améliorer la qualité de l'éducation à partir du savoir et l'expérience des apprenants et des enseignants.

2. L'Unesco encourage l'éducation bilingue et/ou multilingue à tous les niveaux de l'éducation, en tant que moyen de promouvoir l'égalité sociale et entre les sexes, et en tant qu'élément essentiel de sociétés linguistiquement diverses.

3. L'Unesco encourage la démarche qui fait de la langue une composante essentielle de l'éducation interculturelle, en vue d'encourager la compréhension entre différentes populations et d'assurer le respect des droits fondamentaux. 
12 On ne peut nier la cohérence des positions et des actions prises par l'organisation onusienne. La résistance aux dérives de la mondialisation s'y est montrée à la fois lucide, déterminée et constructive.

Le point d'orgue y sera donné à l'occasion d'un long combat, qui débouche en novembre 2005, après un vote quasi-unanime (les Etats-Unis et Israël votant contre, l'Australie, le Nicaragua, le Honduras et le Liberia s'abstenant), sur la «Convention sur la protection et la promotion de la diversité et des expressions culturelles », qui rendait universelle la diversité culturelle.

14 C'est à l'évidence une grande victoire à mettre à l'actif des responsables de la Francophonie. Le texte est un instrument juridique international contraignant. Entré en vigueur depuis mars 2007, il met la culture à l'abri des lois du marché, donc des entraves redoutées de l'OMC et a fortiori des "conditions particulières » propres aux traités bilatéraux (principe de non-subordination aux traités antérieurs).

$-U E$

15 C'est en 2005 également que l'Union Européenne - plutôt muette sur les langues, sujet tabou abandonné au Conseil de l'Europe jusque-là - va soudain rompre un silence devenu étourdissant, celui de sa règle non écrite : respect du multilinguisme officiel.

Un réveil en fanfare sous la forme d'un document fondamental et lucide: Un nouveau cadre stratégique pour le multilinguisme (COM 2005). On s'y refuse à l'unilinguisme anglais : «l'anglais ne suffit pas». Le multilinguisme y est présenté comme une "spécificité de l'UE [...] essentielle à son bon fonctionnement [...], un prix [...], soit 2,28€ par citoyen et par an [...] mais sans lui, une UE démocratique et transparente ne serait pas possible ».

Après un demi-siècle de demi-sommeil, un réveil absolu !

L’Europe passait de la subsidiarité au plurilinguisme.

\section{***}

Il aura fallu un siècle pour reconnaître la nécessité d'un passage de l'Un au Multiple dans le domaine culturel.

Un siècle pour amener les responsables de la politique culturelle à concrétiser officiellement dans des textes tout un ensemble de données simples (pour ne pas dire élémentaires) que leur fournissaient les scientifiques, à savoir :

- l'importance de la langue sur le développement de l'individu

- le rôle de la langue du milieu en matière de scolarisation, d'intégration sociale et de développement socio-économique, politique et culturel de toute société

- l'intérêt du plurilinguisme, tant pour les États que pour les individus

- le rôle majeur de la culture sur le développement économique.

Combien de temps faudra-t-il encore pour observer sur le terrain les conséquences de cette évolution, en d'autres termes, pour passer du de jure au de facto ? Et au-delà, pour que le changement de paradigme ait levé toutes les contradictions de l'unité dans la diversité?

Question interpellante pour la responsabilité de chacun. 


\section{Le français en crise?}

Parmi les causes intrinsèques du recul du français, j’ai relevé (Renard, 2011, 167sv.) (a) l'indifférence de l'opinion publique française à la question de l'avenir de la langue, indifférence certes fort répandue dans le monde, mais dangereuse pour les langues menacées ou en perte de vitesse ; (b) l'attitude laxiste des élites, une inconscience frisant, chez certains, la trahison des clercs ; (c) la désertion affairiste, qui recompose délibérément le monde par un clivage socio-politique; (d) le jacobinisme linguistique de la France, compréhensible à l'époque de la fondation de la République une et indivisible, mais injustifiable aujourd'hui ; (e) son conservatisme normatif, ringard, appauvrissant, et très préjudiciable à la diffusion, parce qu'il entretient la représentation sacralisante et sophistiquée d'une langue peu pragmatique, difficile d'accès; (f) l'incohérence de sa politique linguistique, totalement irréaliste aux plans régional, européen, mondial, et qui trop longtemps l'a empêchée de sortir d'une logique d'unilinguisme - ce qu'elle a fait, malgré tout, avec la signature (mais pas encore la ratification) de la Charte européenne des langues régionales et minoritaires ; (g) la marginalisation de fait des "partenaires" de l'espace francophone, partagés entre la lucidité et la docilité ; (h) la longue inadaptation du cadre institutionnel de la Francophonie à l'importance de l'enjeu; (i) le désintérêt pour les langues nationales partenaires : le refus de respecter le droit élémentaire de tout enfant de se développer dans la langue de son milieu explique pour une grande part l'échec de la scolarisation dans l'Afrique dite francophone, avec les conséquences que cela implique sur le développement sociétal et économique, et sur la diffusion du français.

- Atouts

24

le français dispose d'atouts non négligeables.

C'est d'abord une langue en état. Sa réputation méritée de clarté est liée à une longue tradition de précision textuelle ; mais au-delà de la volonté cultivée de clarifier le sens des 
mots, cette renommée tient davantage à la qualité et l'influence des messages qu'elle a portés. Car il ne faut pas confondre langue et message : les valeurs portées par une langue ne sont pas inscrites dans cette langue (Meschonnic, 1997). Simplement, grâce au niveau de normalisation auquel il est parvenu, le français peut aujourd'hui, - et il n'est pas le seul à le pouvoir, - permettre à des esprits clairs de produire des messages clairs. Soutenu par des efforts appréciables d'instrumentalisation, notamment en néologie et en terminologie, le français est tout à fait apte à contribuer au développement de l'esprit scientifique et critique. Les principaux opérateurs de la Francophonie ont pris au sérieux le défi des inforoutes, notamment par la mise en place de structures qui offrent à la communauté francophone une palette chaque jour plus abondante des produits de l'ingénierie linguistique. Le rééquilibrage des contenus sur la toile est déjà notable.

En outre, la langue française bénéficie d'un imaginaire positif. Ceci est un atout, car les représentations déterminent les pratiques. Dans l'imaginaire de nombreux peuples, le français porte des valeurs de liberté, d'ouverture, de tolérance, de justice, de solidarité et de démocratie, de laïcité de l'Etat, et aussi de culture de la culture.

Ce qui est sans doute le maître atout devait être mis en évidence : la constitution d'une francophonie plurielle, espace ouvert d'échanges culturels et de solidarité entre des peuples différents qui ont choisi le français comme instrument privilégié d'accès à la modernité. Cet atout, peu apprécié à sa juste valeur jusqu'ici dans l'Hexagone, pourrait bien s'y voir valorisé, comme nous le verrons bientôt.

\section{Une conjoncture internationale inquiétante}

La conjoncture internationale est en effet devenue inquiétante.

- La globalisation

En cause, les dérives de plus en plus visibles de la mondialisation. Celle-ci, sous sa forme "globalisation", consacre et renforce la financiarisation de l'économie et la dictature intégriste du profit avec, entre autres effets dévastateurs : la délégitimation du politique, de l'Etat protecteur et de la démocratie, le détricotage des conquêtes sociales et des services publics (là où il $\mathrm{y}$ en a), la dilapidation marchandisée du patrimoine naturel, l'accroissement des inégalités, l'absence d'une régulation internationale fondée sur la justice, l'équité et le respect des droits humains les plus élémentaires, la privatisation de la vie scientifique, sociale et culturelle, la diffusion par des médias toujours plus sophistiqués et incontrôlables d'une pseudo-culture planétaire uniformisée, débile, médiocre et avilissante. Avec, en perspective, les effets prévisibles de révolutions environnementales et génétiques qui risquent de rouvrir de nouveaux débats sur la nature humaine.

Tout cela avec la complicité d'intellectuels peu regardants de l'intérêt général et, dans les Etats considérés comme avancés démocratiquement, - les plus "modernes», - la grandissante soumission d'élus à un pouvoir qui les dépasse.

- La perte des valeurs

$30 \mathrm{Au}$ renversement des valeurs et à leur remplacement par un matérialisme de bas étage déniant toute norme transcendante, se substituent de plus en plus de jeux et de moins en moins de pain. Des masses grandissantes, en manque spirituel, se réfugient avec une crédulité déconcertante dans l'absolutisation de leurs croyances (je vise, entre autres, les diverses sectes qui contaminent le monde chrétien). En outre (ce n'est en rien 
incompatible), ces populations sont conviées à une croisade mondiale contre un prétendu « axe du mal », tout prêt au « choc des civilisations ».

31 Avec, pour corollaires, dans le monde occidental, la mort des idéologies, l'absence de tout projet fiable de société, la domination d'une vision de la modernité symbolisée par la pensée unique, - en réalité absence de pensée.

Et pendant ce temps, que se passe-t-il dans l'autre monde autrefois dénommé «sousdéveloppé ", puis " en voie de développement », aujourd'hui «moyennement avancé », dans cet immense espace où vivent notamment plus d'un milliard de musulmans? Là aussi, la globalisation a ses laissés-pour-compte, victimes de l'ignorance, de la pauvreté, de l'exploitation, de la misère, de l'inégalité, de l'injustice, voire de l'humiliation.

- L'enjeu : le vivre ensemble

L'interdépendance croissante de groupes et de communautés de cultures différentes, amenées à construire une société commune, engendre le plus souvent des replis voire des conflits identitaires, alors que ce pluralisme devrait permettre de mieux vivre la destinée partagée.

Pour parvenir à gérer l'immense brassage auquel nous assistons aujourd'hui, de populations soucieuses à juste titre de protéger leur identité, le remède est connu:il consiste en une régulation institutionnelle, un dispositif sociétal, épanouissant et pacificateur, qui porte le nom de laïcité.

Il s'agit d'établir des règles de "vivre ensemble» garantes d'une coexistence harmonieuse. Il s'agit d'accommoder la liberté de chacun aux croyances individuelles. Pour cela, faire admettre à chacun que ses convictions lui sont propres, qu'elles relèvent de son intimité, qu'il a certes le droit de les professer, mais que ceux qui pensent autrement ont les mêmes droits que lui. Qu'en tout état de cause, la société n'a ni le droit d'interdire ni celui d'imposer une conviction. Pas de religion d'Etat donc, mais une garantie de respect de toutes les options religieuses ou philosophiques.

Vivre ensemble dans la « maison commune ». Certes. Mais la question véritable est de bien vivre ensemble.

Une chose est certaine. L'évolution du monde, inquiétante à bien des égards, suscite l'émergence d'un refus populaire et citoyen de mieux en mieux organisé, qui se traduit par la naissance d'une opinion publique universelle animée d'une extraordinaire demande éthique en faveur d'une autre mondialisation, d'un autre développement planétaire.

Face à ce mouvement grandissant, la Francophonie, de par sa composition, sa diversité et ses valeurs, est tout indiquée pour servir de relais auprès des grandes organisations internationales censées être des instances de régulation. Culturellement, la Francophonie est déjà une forme de mondialisation positive, un laboratoire de dialogue et de solidarité axé, non sur le profit et l'exploitation, mais sur les valeurs humaines. À elle de saisir l'occasion.

\section{Et le français dans tout cela?}

Aujourd'hui, le français est certes une langue internationale, mais parlée par des francophones répandus sur les cinq continents, qui ne sont plus majoritairement Français. 
40 Autrement dit, la France n'est plus propriétaire exclusive de sa langue et donc maitrisant, régentant sa norme. Ceci explique l'assouplissement de celle-ci, d'ailleurs fortement édulcorée dans l'Hexagone, compte tenu de la montée des régionalismes et de l'importance métissante des populations immigrées.

Quel peut donc être le rôle du français?

41 Le français est diffusé partout dans le monde. Ceci signifie que l'on peut trouver dans le monde des foyers de résistance à la perte des valeurs, d'entretien des valeurs universelles...

Sa voie est dès lors toute tracée.

42 Au-delà de permettre à ceux dont la langue n'a pas encore atteint le niveau de développement approprié d'assurer l'accès au monde de la modernité, le français peut amener des esprits clairs à produire des messages clairs dans leur propre langue, de transposer dans celle-ci ces valeurs universelles qui, pour être vraiment universelles, ont besoin d'être exprimées et vécues par tous, quelle que soit leur langue.

43 Langue en partage d'un vaste espace multiculturel, le français peut se vouer à la transmission de valeurs universelles, celles-là même qui transcendent les cultures.

44 Et il semble bien que la politique française prenne cette voie. Dans un article de $2002^{2}$, Xavier North, Délégué général à la langue française et aux langues de France, constatant que "l'infiltration du français dans un grand nombre de cultures est l'une des caractéristiques de la francophonie », invite à " dépasser l'opposition entre le centre et la périphérie, non seulement parce que le centre partage avec la périphérie le dénominateur commun de la pluralité, mais parce que la déterritorialisation des usages linguistiques rend obsolète la notion même de centre » (p. 122).

45 La politique des langues est ainsi devenue, selon cet important fonctionnaire, une "politique des relations entre les langues » (Ibid, 123) avec, pour le français, une fonction médiatrice. La force de la francophonie, conclut-il, est de «pouvoir partager l'extraordinaire richesse offerte par sa diversité » (Ibid, 124).

46 Partager. Ceci implique la réciprocité. On ne peut douter du bénéfice réciproque qu'engendrerait ce partage proposé. S'il l'était sous le signe de l'ouverture, de la tolérance et du respect de l'autre.

47 Toutes les cultures disposent d'un fonds de richesses enfoui dans la tradition, inscrit dans l'inconscient collectif, et qu'un contact privilégié peut réveiller. Lorsque l'échange touche au sens profond des choses, à la vie, à la condition humaine, les valeurs abandonnent leur caractère abstrait pour se fondre dans la réalité colorée du contexte. Et c'est tout profit pour chacun, car il s'enrichit de la différence de l'autre. Ceci n'ôte rien à l'identité, mais à l'inverse précise, conforte celle-ci, l'élève tout en le purifiant ; l'altérité est le révélateur de l'identité, on ne le répétera jamais assez.

48 Les cultures sont évolutives car perméables. Aucune n'est universelle, mais toutes véhiculent des valeurs universalisables. Toutes représentent une manière différente de comprendre le monde et par là constituent notre patrimoine universel.

49 Aucune ne pouvant prétendre déchiffrer le sens profond des choses, leur dialogue, que la tolérance et l'ouverture permet de promouvoir, aide à s'en rapprocher.

50 Dans l'univers interconnecté en train de se construire, les individus doivent se prémunir d'une conception exclusive de la culture. Il faut les éduquer à la distanciation par rapport 
aux évidences ${ }^{3}$, à la désabsolutisation de l'absolu des convictions, à l'éducation de l'esprit critique.

51 Concluant son article de 2007, Xavier North va plus loin. Au moment où se joue dans le monde une lutte décisive d'influence, le français peut devenir cette langue de « contrepouvoir » dont parlait jadis Lionel Jospin, et dans laquelle s'expriment « la résistance à la standardisation culturelle, le refus de l'affadissement des identités, la liberté pour chacun de créer et de s'exprimer dans sa propre culture » (pp. 15-16) ${ }^{4}$.

La dynamique proposée implique l'éradication de toute instrumentalisation politique ou religieuse et une focalisation sur les droits de l'homme et la démocratie, associée à la séparation du temporel et du spirituel.

Bref, en ce domaine, capital pour le "vivre ensemble», la langue française a incontestablement des atouts et elle peut contribuer à " humaniser la mondialisation », objectif de la «stratégie à moyen terme » de l'UNESCO !

\section{Incidences pédagogiques} historiens, philosophes, essayistes, linguistes, éducateurs qui ont illustré, chacun dans leur domaine, les lettres françaises. A l'évidence, les plus vulgarisateurs devront être privilégiés.

- Le dialogue interculturel

Il s'agit d'amener l'apprenant à l'aptitude à appréhender le socle de "bien commun ", local et mondial, fondement de la volonté de vivre ensemble.

61 partager la connaissance de l'Autre. Citons, au hasard, pour un premier niveau de langue : l'analyse de BD étrangères, rendues muettes, l'élaboration d'un projet de voyage ou d'un échange scolaire; pour un niveau plus avancé: l'analyse de contes, de proverbes 
étrangers traduits, des jeux de rôles, la recherche des préjugés dans différents textes, des tables rondes à objectif argumentatif...

- La pensée par l'esprit critique d'unité au profit de celle de la diversité, le rôle du français se doit de prendre une nouvelle dimension, que justifie d'ailleurs une conjoncture internationale devenue inquiétante. 


\section{BIBLIOGRAPHIE}

CALVET, Louis-Jean (1999). Pour une écologie des langues du monde. Paris : Plon.

COMMISSION DES COMMUNAUTÉS EUROPÉENNES (2005). Un nouveau cadre stratégique pour le

multilinguisme. Bruxelles : [COM2005].

MESCHONNIC, Henri (1997). De la langue française. Essai sur une clarté obscure. Paris : Hachette.

NORTH, Xavier (2002). « Le français au défi de la pluralité », Géopolitique africaine, 44.

NORTH, Xavier (2007). « Territoires de la langue française », Hérodote, 126, p. 9-16. DOI : 10.3917/

her.126.0009.

ROCHEBLOINE, François (2014). Rapport d'Information sur la Francophonie : action culturelle, éducative et économique, Rapport $n^{\circ}$ 1723, Assemblée Nationale française.

RENARD, Raymond (2010). « Changement de paradigme culturel : de l'Un au Multiple », in Lorraine Baqué, Marta Estrada, éds, La langue et l'être communiquant. Hommage à Julio Murillo. Mons : CIPA.

RENARD, Raymond (2009). « Laïcité, valeur universalisable de la francophonie. Implications pour l'éducation ", Revue japonaise.

RENARD, Raymond (2014). Pour une laïcité universalisable, 4 éd., Mons : CIPA.

RENARD, Raymond (2010). Structuro-global et verbo-tonal : Variations 1962-2010, Essai de didactique des langues. Mons : CIPA.

RENARD, Raymond (2011). Une éthique pour la francophonie. Questions de géopolitique linguistique, $5^{\mathrm{e}}$ éd., revue et augmentée. Mons : CIPA.

UNESCO (1998). Conférence intergouvernementale sur les politiques culturelles pour le développement, Stockholm (Suède), Rapport final.

UNESCO (1953) L'emploi des langues vernaculaires dans l'enseignement (Monographies sur l'éducation de base, 8). Paris.

UNESCO (2003) Section de l'alphabétisation et de l'éducation non formelle, Division de l'éducation de base, L'alphabétisation, source de liberté, table ronde organisée par l'Unesco.

\section{NOTES}

1. Cf. le récent Rapport de l'Assemblée nationale française (2014).

2. «Le français au défi de la pluralité ", Géopolitique africaine, 44, 2002, 117-132.

3. Claire Lejeune suggérait la transcription « éVIDEnce »!

4. «Pour cela, poursuit North, il faut faire de la langue française un élément d'une politique de souveraineté - il faut qu'elle continue de jouer son rôle dans la construction, toujours inachevée, des identités nationales -, mais il faut aussi défendre la communauté sans territoire du français plus que le territoire des francophones, s'ouvrir aux autres communautés linguistiques par une grande politique de la traduction (absolument essentielle en Europe), et accentuer la présence du français sur les réseaux numériques en 
favorisant la constitution de bibliothèques et d'universités virtuelles. C'est à ce prix, sans doute, qu'au cœur de la bataille des idées, en prenant appui sur la «multipolarité » de l'espace francophone, une politique du français ouverte sur le monde peut apporter une réponse positive aux questions posées par la mondialisation ».

\section{RÉSUMÉS}

$\mathrm{Au}$ moment où un nouveau paradigme linguistique consacre l'abandon de la logique d'unité au profit de celle de la diversité, le rôle du français se devait de prendre une nouvelle dimension, que justifie une conjoncture internationale devenue inquiétante. Parlé sur les cinq continents par des francophones plus nombreux que ceux de l'Hexagone, il est devenu une langue de médiation, d'échange, la langue de l'intercompréhension interculturelle, - pour son plus grand enrichissement d'ailleurs. Les incidences sur la didactique sont importantes, tant au niveau de la formation des maîtres qu'à celui des pratiques de classes. Il s'agit notamment de réviser la notion de culture «française » et d'exploiter le dialogue interculturel, en vue, principalement, de développer l'esprit critique et l'enrichissement de la pensée.

At this time of change in the linguistic paradigm in which the logic of a single language is being abandoned in favour of diversity, the role of French has had to take on a new dimension, especially considering the worrying international situation. Spoken on all five continents by more French-speakers than those living in France itself, French has become the language of negotiation, of exchange, of intercultural understanding - and this, moreover, to its own greater enrichment. The consequences for language-teaching are important, both for teacher-training and for classroom activities. The notion of "French" culture must be revised and the intercultural dialogue exploited in order, above all, to develop a critical mind and enrich ways of thinking.

\section{INDEX}

Mots-clés : logique de diversité, langue de médiation, dialogue interculturel, esprit critique, humanisation

Keywords : logic of diversity, negotiation, intercultural dialogue, critical mind, humanisation

\section{AUTEUR}

\section{RAYMOND RENARD}

Université de Mons

raymond.renard[at]umons.ac.be 